\title{
PERIODS FOR FLAT ALGEBRAIC CONNECTIONS
}

\author{
MARCO HIEN
}

\begin{abstract}
In previous work, [10, we established a duality between the algebraic de Rham cohomology of a flat algebraic connection on a smooth quasi-projective surface over the complex numbers and the rapid decay homology of the dual connection relying on a conjecture by C. Sabbah, which has been proved recently by T. Mochizuki for algebraic connections in any dimension. In the present article, we verify that Mochizuki's results allow to generalize these duality results to arbitrary dimensions also.
\end{abstract}

\section{Introduction}

In [10, we proved a duality theorem for the algebraic de Rham cohomology of a flat algebraic connection $(E, \nabla)$ on a smooth quasi-projective surface over the complex numbers assuming a conjecture of C. Sabbah ([23], Conjecture I.2.5.1) on the existence of the so-called good formal structure after birational pullback of meromorphic connections on surfaces. Lately, a proof of Sabbah's Conjecture in the case of algebraic connections (which lie in the main focus of our work) has been achieved by T. Mochizuki (see 19]). Moreover, in another astonishing work 20, T. Mochizuki is able to prove a far reaching higherdimensional generalization of this result, namely the existence of a good lattice again after a suitable birational pull-back (loc.cit., Theorem 19.5). As in the one- and two-dimensional case, the canonical next step into structural examinations of flat meromorphic connections consists in the question of lifting the good formal properties to asymptotic analogues. This step is also carried out in Mochizuki's paper [20] and results in the construction of Stokes structures for flat meromorphic connections in arbitrary dimensions. With these tools in hand, we are now able to construct the period pairing of a flat algebraic connection between the algebraic de Rham cohomology and the rapid decay homology which we introduced in [10] and prove the perfectness of this period pairing unconditionally in all dimensions. As a consequence, assuming rational structures of the given geometric data over a subfield $k \subset \mathbb{C}$ as well as of the local system associated to the flat connection over another subfield $F \subset \mathbb{C}$, we deduce a well-defined notion of the period determinant arising from the comparison of these rational structures by means of the perfect period pairing. This determinant will be an element in the quotient $\mathbb{C}^{\times} / k^{\times} F^{\times}$. One of the motivations to study this determinant lies in the mysterious analogies between flat connections over the complex numbers and $\ell$-adic sheaves on varieties over finite fields, according to which the period determinant is expected to behave as the $\varepsilon$-factor in the latter theory. If one restricts to the subcategory of regular singular connections, corresponding to the tamely ramified sheaves over finite fields, the period determinant and its analogies to the $\varepsilon$-factor has been extensively studied by T. Saito and T. Terasoma in [26]. With the present work, we hope to contribute to a generalization of these lines of thought to the irregular singular case.

Let us give a short summary of the contents of this paper. The main object under consideration is the period pairing between the algebraic de Rham cohomology of the given flat algebraic connection and the rapid decay homology - analytic in nature - of the dual connection. We will discuss this construction in section 5. The main result Theorem 5.2, the perfectness of the period pairing, relies on a local duality between the algebraic de Rham complex and an analytic de Rham complex of the dual bundle with asymptotically flat coefficients. This duality will be proved in Theorem 4.4 and Proposition 5.1 compares the asymptotically flat de Rham cohomolgy with the rapid decay homology creating the link between the local duality and the period pairing.

1991 Mathematics Subject Classification. 14F40, 14F10.

Key words and phrases. Period integrals, meromorphic connections, algebraic de Rham cohomology. 


\section{Formal completion and real oriented blow-up}

We consider the following geometric situation. Let $U$ be a smooth quasi-projective variety over $\mathbb{C}$ and $X$ a smooth projective variety such that $D:=X \backslash U$ is a divisor with normal crossings. Introducing real polar coordinates around each irreducible component of $D$ leads to the real oriented blow-up $\pi$ : $\widetilde{X} \rightarrow X^{\text {an }}$. Locally at some $p \in D$, we can choose complex coordinates $x_{1}, \ldots, x_{d}$ such that $p=0$ and $D=\left\{x_{1} \cdots x_{k}=0\right\}$. Then $\pi$ reads as

$$
\pi:\left([0, \varepsilon) \times S^{1}\right)^{k} \times Y \rightarrow X^{\text {an }},\left(\left(r_{\nu}, e^{i \vartheta_{\nu}}\right)_{\nu=1}^{k}, y\right) \mapsto\left(\left(r_{\nu} \cdot e^{i \vartheta_{\nu}}\right)_{\nu=1}^{k}, y\right),
$$

where $Y$ is a small analytic neighbourhood of $0 \in \mathbb{C}^{d-k}, y=\left(x_{k+1}, \ldots, x_{d}\right)$ and $\varepsilon>0$ a small positive number. Restricted to $\pi^{-1}\left(U^{\text {an }}\right)$ the real oriented blow-up is a homeomorphism by which we read $U^{\text {an }}$ as a subspace of $\widetilde{X}$. We write $\widetilde{\jmath}: U^{\text {an }} \hookrightarrow \widetilde{X}$ for the inclusion.

On $\widetilde{X}$, we consider the following sheaves of functions (cp. [23, II.1.). Firstly, $\widetilde{X}$ carries the structure of a real manifold with boundary. Additionally, the logarithmic differential operators $\bar{x}_{\nu} \bar{\partial}_{x_{\nu}}$ act on the sheaf $C_{\widetilde{X}}^{\infty}$ of $C^{\infty}$ functions. Let $\mathcal{A}_{\widetilde{X}}$ be the sheaf of functions which in the local siuation as above is given by

$$
\mathcal{A}_{\widetilde{X}}:=\bigcap_{\nu=1}^{k} \operatorname{ker}\left(\bar{x}_{\nu} \bar{\partial}_{x_{\nu}}\right) \cap \bigcap_{\nu=k+1}^{d} \operatorname{ker} \bar{\partial}_{x_{\nu}} \subset C_{\widetilde{X}}^{\infty}
$$

Local sections in $\mathcal{A}_{\widetilde{X}}$ for some open $\Omega \subset \widetilde{X}$ are differentiable functions on $\Omega$ which are holomorphic on $\Omega \cap U^{\text {an }}$ and admit an asymptotic development in the higher-dimensional analogue of Poincaré's asymptotic developments due to Majima (cp. Proposition B.2.1 in [23] and [13]).

Next, if $\mathcal{P}_{\widetilde{X}}^{\leq D}$ denotes the sheaf of $C^{\infty}$-functions on $\widetilde{X}$ which are flat on $\pi^{-1}(D)$, i.e. all of whose derivations vanish on $\pi^{-1}(D)$ (cp. [15]), we consider

$$
\mathcal{A}_{\widetilde{X}}^{<D}:=\mathcal{A}_{\widetilde{X}} \cap \mathcal{P}_{\widetilde{X}}^{<D}
$$

Similarly, the local sections of $\mathcal{A}_{\widetilde{X}}^{<D}$ are holomorphic restricted to $U^{\text {an }}$ and with vanishing asymptotic development (cp. Proposition II.1.1.11 in [23]). More precisely, if $u \in \mathcal{A}_{\tilde{X}}^{<D}(\Omega)$ then for any compact $K \subset \Omega$ and any $N \in \mathbb{N}^{k}$, the function $u$ satisfies

$$
|u(x)| \leq C_{K, N} \cdot\left|x_{1}\right|^{N_{1}} \cdots\left|x_{k}\right|^{N_{k}} \text { for all } x \in K \backslash \pi^{-1}(D),
$$

in terms of local coordinates as above such that locally $D=\left\{x_{1} \cdots x_{k}=0\right\}$.

Due to Lemme II.1.1.18 in [23], we have the following fine resolution of $\mathcal{A}_{\widetilde{X}}^{<D}$

$$
\mathcal{A}_{\widetilde{X}}^{<D} \hookrightarrow\left(\mathcal{P}_{\widetilde{X}}^{<D} \otimes_{\pi^{-1} C_{X}^{\infty}} \pi^{-1} \Omega_{X^{\text {an }}}^{\infty,(0, \bullet)}, \bar{\partial}\right)
$$

where $\Omega_{X^{\text {an }}}^{\infty,(p, q)}$ denotes the sheaf of $C^{\infty}$-forms of degree $(p, q)$ on $X$.

Analogously, if $\mathcal{A}_{\widetilde{X}}^{\bmod D}$ denotes the sheaf of functions on $\widetilde{X}$ which are holomorphic on $U^{\text {an }}$ and of moderate growth along $\widetilde{D}$, we have a fine resolution

$$
\mathcal{A}_{\widetilde{X}}^{\bmod D} \hookrightarrow\left(\mathcal{P}^{\bmod D} \otimes_{\pi^{-1} C_{X}^{\infty}} \pi^{-1} \Omega_{X^{\text {an }}}^{\infty,(0, \cdot)}, \bar{\partial}\right),
$$

with $\mathcal{P}^{\bmod D}$ being the sheaf of $C^{\infty}$-functions on $\widetilde{X}$ with moderate growth at $\pi^{-1}(D)$.

Finally, let $\mathfrak{D} \mathfrak{b}_{\widetilde{X}}^{\text {rd, }-s}$ denote the sheaf of rapid decay distributions on $\tilde{X}$, i.e. the sheaf whose local section on small open $V \subset \widetilde{X}$ are distributions

$$
\varphi \in \mathfrak{D b}_{\widetilde{X}}^{-s}(V):=\operatorname{Hom}_{\text {cont }}\left(\Gamma_{c}\left(V, \Omega_{\widetilde{X}}^{\infty, s}\right), \mathbb{C}\right)
$$

on the space $\Omega_{\widetilde{X}}^{\infty, s}$ of $C^{\infty}$ differential forms on $\widetilde{X}$ of degree $s$ with compact support in $\tilde{X}$ satisfying the following condition: choosing coordinates $x_{1}, \ldots, x_{n}$ on $X$ such that $D \cap V=\left\{x_{1} \cdots x_{k}=0\right\}$, we require 
that for any compact $K \subset V$ and any element $N \in \mathbb{N}^{k}$ there are $m \in \mathbb{N}$ and $C_{K, N}>0$ such that for any test form $\eta$ with compact support in $K$ the estimate

$$
|\varphi(\eta)| \leq C_{K, N} \sum_{i} \sup _{|\alpha| \leq m} \sup _{K}\left\{|x|^{N}\left|\partial^{\alpha} f_{i}\right|\right\}
$$

holds, where $\alpha$ runs over all multi-indices of degree less than or equal to $m$ and $\partial^{\alpha}$ denotes the $\alpha$-fold partial derivative of the coefficient functions $f_{i}$ of $\eta$ in the chosen coordinates.

\section{Good formal lattices and decompositions (after T. Mochizuki)}

\subsection{Deligne-Malgrange lattices}

Let there be given a flat meromorphic connection $\nabla$ on the locally free $\mathcal{O}_{X^{\text {an }}}(* D)$-module $E$ or rank $r$, where $D \subset X$ is a divisor with normal crossings. After chioce of a local trivialization $E \cong\left(\mathcal{O}_{X} \text { an }(* D)\right)^{r}$, the connection reads as $\nabla=d+A$ with the connection matrix $A \in M\left(r \times r, \Omega_{X}^{1}\right.$ an $\left.(* D)\right)$. A change $T \in \mathrm{GL}_{r}\left(\mathcal{O}_{X}\right.$ an $\left.(* D)\right)$ of the trivialization transforms the connection matrix due to the formula

$$
A^{\prime}:=T^{-1} d T+T^{-1} A T .
$$

The local classification of these connections, i.e. of the connection matrices up to this transformation, is a difficult task in general.

The first major subdivision of flat meromorphic connections lies in the distinction between regular singular connections and irregular singular ones. Let us recall this notion in the given geometric situation, i.e. with $D$ being a normal crossing divisor. Then, a flat meromorphic connection $(E, \nabla)$ is regular singular, if there is a trivialization $E \cong\left(\mathcal{O}_{X}(* D)\right)^{r}$ such that the resulting connection matrix has logarithmic poles along $D$ at most, i.e. $A$ can be written as $A=\sum_{i=1}^{k} A_{i}(x) \operatorname{dlog} x_{i}+\sum_{j=k+1}^{d} A_{j}(x) d x_{j}$ with holomorphic matrices $A_{i}(x) \in M\left(r \times r, \mathcal{O}_{X}\right.$ an $)$. The structure of regular singular connections is well understood (see [8]), in particular we know that each regular singular connection is a succesive extension of rank one connections. For the latter, one finds a basis vector $e$ over $\mathcal{O}_{X}$ an such that

$$
\nabla e=e \otimes\left(\lambda_{1} \operatorname{dlog} x_{1}+\ldots+\lambda_{k} \operatorname{dlog} x_{k}\right)
$$

for some $\lambda \in \mathbb{C}^{k}$. Such a connection will be denoted by $x^{\lambda}$.

Next, the most elementary irregular singular connections are rank one connections which in a suitable basis vector $e$ read as $\nabla e=e \otimes d \mathfrak{a}$ for some $\mathfrak{a} \in \mathcal{O}_{X^{\text {an }}}(* D)$, which up to isomorphism depends on $\mathfrak{a} \bmod \mathcal{O}_{X}$ an only. Such a connection will be denoted by $e^{\mathfrak{a}}$.

Now, let $Y \subset D$ be a stratum in the natural stratification of the normal crossing divisor $D$. Passing from $\mathcal{O}_{X}$ an to the formal completion $\mathcal{O}_{\widehat{X \mid Y}}$ of $\mathcal{O}_{X}$ an along $Y$ leads to the problem of formal classification, to which a extensive answer is given in the case $\operatorname{dim} X=1$ by the Levelt-Turrittin Theorem (see [14], Chapter III). In [23, C. Sabbah investigated the two-dimensional situation leading to a precise conjecture as well as partial results in this direction. Recently, T. Mochizuki was able to give a proof Sabbah's Conjecture and a higher-dimensional generalization using a different apporach by exmamining the socalled good lattices which go back to Malgrange (see [16]). We will now explain Mochizuki's result about these formal properties of meromorphic connections. For more details, we refer to [20, chapter 5.

Let us choose local coordinates such that $D=\left\{x_{1} \cdots x_{k}=0\right\}$ with the irreducible components $D_{i}=\left\{x_{i}=0\right\}$ and consider the local situation, i.e. we put $X=\Delta^{k} \times Y$ where $\Delta^{k}$ is s small poly-disc in $\mathbb{C}^{k}$ centered at the origin and $Y$ is a small neighbourhood of the origin in the remaining variables. Accordingly, we will denote the first $k$ variables by $z_{1}, \ldots, z_{k}$ and the others by $y_{1}, \ldots, y_{d-k}$. Let $\leq$ be the partial order on $\mathbb{Z}^{k}$ given by $m \leq n$ if $m_{i} \leq n_{i}$ for all $i$. Now, any meromorphic function $f \in \mathcal{O}_{X}$ an $(* D)$ admits a Laurent expansion $f=\sum_{m \in \mathbb{Z}^{k}} f_{m}(y) z^{m}$ with holomorphic functions $f_{m} \in \mathcal{O}_{Y}$. The order of $f$ will be the minimum

$$
\operatorname{ord}(f)=\min \left\{m \in \mathbb{Z}^{k} \mid f_{m} \neq 0\right\},
$$

assuming that this minimum with respect to $\leq$ exists. T.Mochizuki defines the notion of a good set of irregular values on $(X, D)$ to be a finite set $S \subset \mathcal{O}_{X^{\text {an }}}(* D) / \mathcal{O}_{X^{\text {an }}}$, such that

i) $\operatorname{ord}(\mathfrak{a})$ and $\operatorname{ord}(\mathfrak{a}-\mathfrak{b})$ exist for all $\mathfrak{a} \neq \mathfrak{b}$ in $S$, 
ii) the set $\{\operatorname{ord}(\mathfrak{a}-\mathfrak{b}) \mid \mathfrak{a}, \mathfrak{b} \in S\}$ is totally ordered with respect to $\leq$ on $\mathbb{Z}^{k}$,

iii) the leading terms $\mathfrak{a}_{\operatorname{ord}(\mathfrak{a})}(p)$ and $(\mathfrak{a}-\mathfrak{b})_{\operatorname{ord}(\mathfrak{a}-\mathfrak{b})}(p)$ of the Laurent expansions are non-vanishing for all $p \in Y$.

For any subset $I \subset\{1, \ldots, k\}$, let $I^{c}$ be its complement and furthermore $D_{I}:=\bigcap_{i \in I} D_{i}$ and $D(I):=$ $\bigcup_{i \in I} D_{i}$. The completion of $X$ along $D_{I}$ resp. $D(I)$ will is denoted by $\widehat{D}_{I}$ resp. $\widehat{D}(I)$. For a given subset $S \subset \mathcal{O}_{X^{\text {an }}}(* D) / \mathcal{O}_{X^{\text {an }}}$ let $S(I):=\left\{\mathfrak{a} \bmod \mathcal{O}_{X^{\text {an }}}\left(* D\left(I^{c}\right)\right) \mid \mathfrak{a} \in S\right\}$

Let $\mathbb{E}$ be a lattice in $E$, i.e. a locally free $\mathcal{O}_{X}$ an-module such that $\mathbb{E} \otimes \mathcal{O}_{X^{\text {an }}}(* D)=E$. Then $\mathbb{E}$ is called an unramified good lattice at $p \in D$ if there is a good set of irregular values $S$ as above such that for each $I$ we have a formal decomposition

$$
\left.(\mathbb{E}, \nabla)\right|_{\widehat{D}_{I}}=\bigoplus_{\mathfrak{a} \in S(I)}\left({ }^{I} \widehat{\mathbb{E}_{\mathfrak{a}}},{ }^{I} \widehat{\nabla_{\mathfrak{a}}}\right)
$$

such that

$$
\left(\widehat{\nabla_{\mathfrak{a}}}-d \mathfrak{a}\right)\left({ }^{I} \widehat{\mathbb{E}_{\mathfrak{a}}}\right) \subset{ }^{I} \widehat{\mathbb{E}_{\mathfrak{a}}} \otimes\left(\Omega_{X^{\text {an }}}^{1}(\log D(I))+\Omega_{X^{\text {an }}}^{1}\left(* D\left(I^{c}\right)\right)\right) .
$$

The set $S$ of irregular values is uniquely determined by the given connection and hence it is denoted by $S:=\operatorname{Irr}(\nabla)$.

The given connection $(E, \nabla)$ is said to admit a good lattice at $p \in D$, if for some coordinate neighbourhood $U=\Delta^{d}$ of $p$ as above, there exists a ramification map

$$
\rho_{e}: \Delta^{d} \rightarrow U,\left(t_{1}, \ldots, t_{d}\right) \mapsto\left(t_{1}^{e}, \ldots, t_{k}^{e}, t_{k+1}, \ldots, t_{d}\right)
$$

for some $e \in \mathbb{N}_{0}$ such that $\rho_{e}^{*}(E, \nabla)$ admits an unramified good lattice $\widetilde{\mathbb{E}}$. With these notions, T. Mochizuki proves the following far reaching result

Theorem 3.1 (T. Mochizuki, [20, Theorem 19.5). Let $(E, \nabla)$ be a flat meromorphic connection on $(X, D)$. Then there exists a regular birational map $\varphi: X^{\prime} \rightarrow X$ such that the pull-back $\varphi^{*}(E, \nabla)$ admits a good lattice at any $p \in \varphi^{-1}(D)$.

Actually, the theorem in [20] is more precise in the sense that due to Malgrange's work ([16]) one knows that generically there is a "canonical lattice", which Mochizuki calls the Deligne-Malgrange lattice. Mochizuki proves that its extension to the whole $X$ is again locally free over $\mathcal{O}_{X}$ an and a good lattice.

\subsection{Good decomposition in multisectors}

If we apply Mochizuki's Theorem 3.1 to the given flat meromorphic connection $(E, \nabla)$ on $(X, D)$, we know that the pull-back with respect to some birational map and some finite ramification admits a good lattice $\mathbb{E}$. It will be important for our purposes to see that the resulting formal decomposition can be lifted to an asymptotic one on small multisectors. In dimension two, this is the ingredient of [23, II.2, in higher dimensions it can be derived from Mochizuki's approach of good lattices and can be found in [20] also, see the remark after the theorem below.

Let $\mathcal{A}_{\widetilde{X} \mid Z}$ denote the formal completion of $\mathcal{A}_{\widetilde{X}}$ along $\pi^{-1}(Z)$ for a closed subset $Z \subset X$ and $T_{Z}$ the natural morphism $\mathcal{A}_{\widetilde{X}} \rightarrow \mathcal{A}_{\widetilde{X} \mid Z}$. We will consider mainly the case where $Z \subset D$ is a union of local irreducible components of $D$. Note that $\left.\mathcal{A}_{\widehat{X} \mid Z}\right|_{\pi^{-1}(Z)}=\pi^{-1} \mathcal{O}_{\widehat{X \mid Z}}$ in such a case. The following generalization of the one-dimensional Borel-Ritt theorem tells us that the sequence $0 \rightarrow \mathcal{A}_{\widetilde{X}}^{<D} \rightarrow \mathcal{A}_{\widetilde{X}} \stackrel{T_{P}}{\rightarrow}$ $\mathcal{A}_{\widehat{X} \mid D} \rightarrow 0$ is exact (see [23, II.1.1.16). The formal decompositions (3.1) given by the existence of an unramified good lattice can indeed be lifted in the follwing sense:

Theorem 3.2 (T. Mochizuki, [20]). Let (E, $\nabla)$ be a flat meromorphic connection and assume that it admits an unramified good lattice $\mathbb{E}$. Let $\vartheta \in \pi^{-1}(D)$ be any multi-direction. Then there exists a small mutlisector $S \subset X \backslash D$ around $\vartheta$ with closure $\bar{S} \subset \widetilde{X}$ such that we have a $\nabla$-flat decomposition

$$
\left.\left(\pi^{*} \mathbb{E} \otimes_{\pi^{*} \mathcal{O}_{X} \text { an }} \mathcal{A}_{\tilde{X}}\right)\right|_{\bar{S}}=\bigoplus_{\mathfrak{a} \in \operatorname{Irr}(\nabla)} \mathcal{G}_{\mathfrak{a}, S},
$$

where the connection on $\mathcal{G}_{\mathfrak{a}, S}$ induced by $\nabla-d \mathfrak{a}$ is logarithmic, hence regular singular. 
The proof of Theorem 3.2 is completely contained in [20. However, since Mochizuki develops the theory of Stokes structures in a more general setting, adapted to the examination of wild twistor $\mathcal{D}$ modules, and in much more detail than needed here, the proof of the above theorem is not so easily found in loc.cit. It occupies several steps and culminates in the statement to be found in Remark 7.73, [20]. We plan to include a short overview of the necessary steps in a future version of this paper.

\section{The local duality pairing}

Our aim now is to study the meromorphic de Rham complex associated to the flat algebraic connection $(E, \nabla)$ by lifting to the real oriented blow-up $\pi: \widetilde{X} \rightarrow X^{\text {an }}$. To this end, we consider the following de Rham complexes on $\widetilde{X}$ :

Definition 4.1. The asymptotically flat de Rham complex is defined to be the complex

$$
\operatorname{DR}_{\widetilde{X}}^{<D}\left(\nabla^{\vee}\right):=\mathcal{A}_{\widetilde{X}}^{<D} \otimes_{\pi^{-1}\left(\mathcal{O}_{X^{\text {an }}}\right)} \pi^{-1}\left(\operatorname{DR}_{X^{\operatorname{an}}}\left(\nabla^{\vee}\right)\right) \in D^{b}\left(\mathbb{C}_{\tilde{X}}\right)
$$

and the moderate de Rham complex to be the complex

$$
\left.\operatorname{DR}_{\widetilde{X}}^{\bmod D}(\nabla):=\mathcal{A}_{\widetilde{X}}^{\bmod D} \otimes_{\pi^{-1}\left(\mathcal{O}_{X}\right. \text { an }}\right) \pi^{-1}\left(\operatorname{DR}_{X^{\operatorname{an}}}(\nabla)\right) \in D^{b}\left(\mathbb{C}_{\widetilde{X}}\right) .
$$

The moderate de Rham complex computes the meromorphic (and hence also the algebraic) de Rham cohomology of $(E, \nabla)$ (cf. [23], Corollaire 1.1.8):

$$
\mathbb{H}^{k}\left(\widetilde{X}, \mathrm{DR}_{\widetilde{X}}^{\bmod D}(\nabla)\right)=H_{d R}^{k}(U, E, \nabla)
$$

Since multiplication gives a map $\mathcal{A}_{\widetilde{X}}^{\bmod D} \otimes_{\mathbb{C}} \mathcal{A}_{\widetilde{X}}^{<D} \rightarrow \mathcal{A}_{\widetilde{X}}^{<D}$, we deduce that the usual wedge product of a differential form with moderate growth and an asymptotically flat differential form is again asymptotically flat. This leads to the following

Definition 4.2. The local duality pairing is the natural pairing

$$
\operatorname{DR}_{\widetilde{X}}^{\bmod D}\left(\nabla^{\vee}\right) \otimes_{\mathbb{C}} \mathrm{DR}_{\widetilde{X}}^{<D}(\nabla) \rightarrow \mathrm{DR}_{\widetilde{X}}^{<D}\left(\mathcal{O}_{X}, d\right)
$$

induced by the wedge product of forms and the natural contraction $E \otimes E^{\vee} \rightarrow \mathcal{O}_{X}$ of the vector bundle $E$ and its dual to the trivial line bundle.

Before stating the local duality result, we want to prove that the complexes involved are concentrated in one degree whenever $(E, \nabla)$ admits a good lattice on $(X, D)$.

Proposition 4.3. If $(E, \nabla)$ admits a good lattice on $(X, D)$, the complexes $\operatorname{DR}_{\widetilde{X}}^{\bmod D}(\nabla)$ and $\mathrm{DR}_{\widetilde{X}}^{<D}(\nabla)$ have cohomology in degree zero only.

Proof. Due to Mochizuki's results, Theorem 3.1 and Theorem 3.2 above, there exists a bicyclic ramification $\rho: Y \rightarrow X$ such that locally on $\widetilde{Y}$ the pull-back connection $\rho^{-1}(\nabla)$ admits a decomposition (3.2) for small enough multisectors $S$. Let $\pi_{X}: \widetilde{X} \rightarrow X^{\text {an }}$ and $\pi_{Y}: \widetilde{Y} \rightarrow Y^{\text {an }}$ denote the oriented real blow-up of $Z:=\rho^{-1}(D)$ and $D$ respectively. The projection formula for the lift $\widetilde{\rho}: \widetilde{Y} \rightarrow \widetilde{X}$ gives

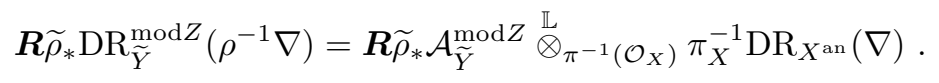

Now, $\widetilde{\rho}$ being a finite map and since obviously $\boldsymbol{R} \widetilde{\rho}_{*} \mathcal{A}_{\widetilde{Y}}^{\bmod Z}=\mathcal{A}_{\widetilde{X}}^{\bmod D}$ (using the resolution (2.3)), it follows that it suffices to prove the claim on $\tilde{Y}$. Hence, we can assume that we have the decompositions 3.2 for the connection $(E, \nabla)$ locally on $\tilde{X}$. Since the decomposition is $\nabla$-flat, we can further restrict to the case of one summand $\mathcal{G}_{\mathfrak{a}, S}$, i.e. we only have to consider the case where a priori $(E, \nabla)$ is of the form $(E, \nabla)=e^{\mathfrak{a}} \otimes R_{\mathfrak{a}}$ for some regular singular connection $R_{\mathfrak{a}}$. Since every regular singular connection is a successive extension of regular singular line bundles, we can further reduce to the case $R_{\mathfrak{a}}=x^{\lambda}$ with a $\lambda \in \mathbb{C}^{d}$.

The proof will now follow the same arguments in the asymptotically flat as well as in the moderate case. Since it is a local statement, we restrict to the local situation at some point $x_{0} \in D=\left\{x_{1} \cdots x_{k}=0\right\}$. 
Let $\vartheta \in \pi^{-1}\left(x_{0}\right) \simeq\left(S^{1}\right)^{k}$ be a direction in $\widetilde{D}$ over $x_{0}$. Then the complex of stalks at $\vartheta$ which we have to consider is given as

$$
\ldots \longrightarrow\left(\mathcal{A}_{\tilde{X}}^{? D_{t}} \otimes_{\pi^{-1} \mathcal{O}_{X}} \pi^{-1} \Omega_{X}^{p}\right)_{\vartheta} \stackrel{\nabla}{\longrightarrow}\left(\mathcal{A}_{\tilde{X}}^{? D} \otimes_{\pi^{-1} \mathcal{O}_{X}} \pi^{-1} \Omega_{X}^{p+1}\right)_{\vartheta} \longrightarrow \ldots
$$

where ? stands for either $<$ or mod. In degree $p$ and with the usual basis $d x_{I}$ for $I=\left\{1 \leq i_{1}<\ldots<\right.$ $\left.i_{p} \leq d\right\}$ of $\Omega_{X}^{p}$, the connection map $\nabla$ in (4.3) reads as

$$
\sum_{\# I=p} w_{I} d x_{I} \mapsto \sum_{\# J=p+1}\left(\sum_{j \in J} \operatorname{sgn}_{J}(j)\left(Q_{j} w_{J \backslash\{j\}}\right)\right) d x_{J}
$$

with

$$
Q_{j} u:=\frac{\partial}{\partial x_{j}} u+\frac{\partial \mathfrak{a}}{\partial x_{j}} \cdot u+x_{j}^{-1} \lambda_{j} \cdot u
$$

and where we define $\operatorname{sgn}_{J}(j):=(-1)^{\nu}$ for $J=\left\{j_{1}<\ldots<j_{p+1}\right\}$ and $j_{\nu}=j$.

Consider a germ of a section $\omega$ of $\mathcal{A}_{\widetilde{X}}^{? D_{t}} \otimes \pi^{-1} \Omega_{X_{t}}^{p+1}$,

$$
\omega=\sum_{\# J=p+1} w_{J} d x_{J}
$$

such that $\nabla \omega=0$. We have to find a $p$-form $\eta$ with appropriate growth condition such that $\nabla \eta=\omega$.

To achieve this, let $s \in \mathbb{N}$ be an integer such that $w_{J}=0$ for all $J$ with $J \cap\{1, \ldots, s-1\} \neq \emptyset$ (which is an empty condition for $s=1$ ). We prove that we can find a $p$-form $\eta$ with coefficients in $\mathcal{A}_{\tilde{X}}^{? D}$ such that

$$
(\omega-\nabla \eta) \in \sum_{J \cap\{1, \ldots, s\}=\emptyset} \mathcal{A}_{\tilde{X}, \vartheta}^{? D} d x_{J}
$$

The assertion then follows immediately by induction.

By assumption

$$
0=\nabla \omega=\sum_{\# K=p+2}\left(\sum_{k \in K} \operatorname{sgn}_{K}(k)\left(Q_{k} w_{K \backslash\{k\}}\right)\right) d x_{K} .
$$

Taking $k<s$ and $s \in J$ and examining the summand of (4.5) corresponding to a set of the form $K:=\{q\} \cup J$ we see that

$$
Q_{q} w_{J}=0 \text { for all such } q=1, \ldots, s-1 .
$$

Now, consider the system $\left(\Sigma_{J}\right)$ of partial differential equations for the unknown function $u_{J}$, where $J$ is a fixed subset $J \subset\{s, \ldots, d\}$ of cardinality $p+1$ with $s \in J$ :

$$
\left(\Sigma_{J}\right):\left\{\begin{array}{l}
Q_{q} u_{J}=0 \quad \text { for all } q=1, \ldots, s-1 \\
Q_{s} u_{J}=w_{J},
\end{array}\right.
$$

together with the integability assumption (4.6). Systems of this type had been studied by Majima (cf. [13]) before. In the case of $\mathcal{A}_{\widetilde{X}, \vartheta}^{<D}$, the result follows from [24], Appendix A, in the moderate case we refer to [10], Theorem A.1 (which is formulated in dimension two only but generalizes without difficulties to the case of arbitrary dimension).

In each case, we can always find a solution $u_{J} \in \mathcal{A}_{\tilde{X}, \vartheta}^{? D}$ for any such $J \subset\{s, \ldots, d\}$ of cardinality $p+1$ and $s \in J$ and if we let

$$
\eta:=\sum_{J \text { as above }} u_{J} d x_{J}
$$

we easily see that (4.4) is satisfied.

In particular, the proposition applies to the trivial conenction $\left(\mathcal{O}_{x}, d\right)$ for any $(X, D)$ such that $D$ has normal crossings. The only interesting cohomology sheaf is

$$
\mathcal{H}^{0} \operatorname{DR}_{\tilde{X}}^{<D}\left(\mathcal{O}_{X}, d\right)=\widetilde{\jmath}_{!} \mathbb{C}_{U^{\text {an }}},
$$


where $\tilde{\jmath}: U^{\text {an }} \hookrightarrow \widetilde{X}$ again denotes the inclusion. Therefore, the local duality pairing can be written as

$$
\operatorname{DR}_{\widetilde{X}}^{\bmod D}\left(\nabla^{\vee}\right) \otimes_{\mathbb{C}} \operatorname{DR}_{\widetilde{X}}^{<D}(\nabla) \rightarrow \widetilde{\jmath}_{!} \mathbb{C}_{U \text { an }}
$$

We are now ready to state the following local duality theorem which lies in the heart of this work:

Theorem 4.4. The local duality pairing (4.1) is perfect in the sense that the induced morphisms

$$
\operatorname{DR}_{\widetilde{X}}^{\bmod D}\left(\nabla^{\vee}\right) \rightarrow \boldsymbol{R} \operatorname{Hom}_{\widetilde{X}}\left(\operatorname{DR}_{\widetilde{X}}^{<D}(\nabla), \widetilde{\jmath} ! \mathbb{C}\right) \quad \text { and } \quad \operatorname{DR}_{\widetilde{X}}^{<D}(\nabla) \rightarrow \boldsymbol{R} \operatorname{Hom}_{\widetilde{X}}\left(\operatorname{DR}_{\widetilde{X}}^{\bmod D}\left(\nabla^{\vee}\right), \widetilde{\jmath} ! \mathbb{C}\right)
$$

are isomorphisms in the derived category.

We give the proof of this theorem in the next section.

\subsection{Proof of Theorem 4.4}

According to Mochizuki's results, $(E, \nabla)$ admits a good lattice after some regular birational morphism $b: Y \rightarrow X$. Let $Z:=b^{-1}(D)$ be the resulting divisor in $Y$ and let $\pi_{X}: \widetilde{X} \rightarrow X^{\text {an }}$ and $\pi_{Y}: \widetilde{Y} \rightarrow Y^{\text {an }}$ denote the oriented real blow-ups. Let $\widetilde{b}: \widetilde{Y} \rightarrow \widetilde{X}$ be a lift of $b$. We will also use the following notation for the embeddings $\widetilde{\jmath}: X^{\text {an }} \backslash D \hookrightarrow \widetilde{X}$ and $\widetilde{\imath}: Y^{\text {an }} \backslash Z \hookrightarrow \widetilde{Y}$. The de Rham complexes on the real oriented blow-ups behave functorial with respect to this situation:

Lemma 4.5. We have natural isomorphisms

$$
\boldsymbol{R} \widetilde{b}_{*}\left(\mathrm{DR}_{\widetilde{Y}}^{\bmod Z}\left(b^{*} \nabla\right)\right) \cong \mathrm{DR}_{\widetilde{X}}^{\bmod D}(\nabla) \text { and } \boldsymbol{R} \widetilde{b}_{*}\left(\mathrm{DR}_{\tilde{Y}}^{<Z}\left(b^{*} \nabla\right)\right) \cong \mathrm{DR}_{\widetilde{X}}^{<D}(\nabla)
$$

Proof. Via the projection formula - note that $E$ is locally free - we obtain

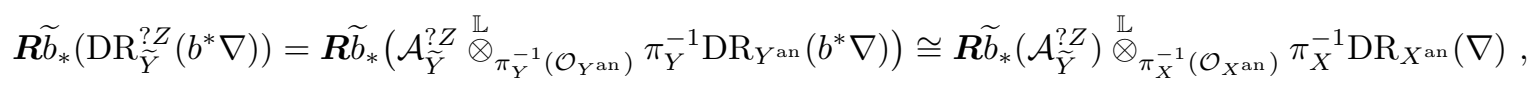

where ? stands for $<$ or mod respectively. Hence, it suffices to prove that $\boldsymbol{R} \widetilde{b}_{*}\left(\mathcal{A}_{\tilde{\widetilde{Y}}}^{? Z}\right)=\mathcal{A}_{\tilde{X}}^{? D}$.

Since the restiction of $b: Y^{\text {an }} \rightarrow X^{\text {an }}$ to $Y^{\text {an }} \backslash Z$ is an isomorphism $Y^{\text {an }} \backslash Z \rightarrow X^{\text {an }} \backslash D=U^{\text {an }}$, we see that the sheaves of flat $C^{\infty}$-functions on each space, i.e. all of whose derivations vanish on the boundary, are compatible with $\widetilde{b}$, i.e.

$$
\widetilde{b}_{*} \mathcal{P}_{\widetilde{Y}}^{<Z}=\mathcal{P}_{\widetilde{X}}^{<D}
$$

Similarily, we have $\widetilde{b}_{*} \mathcal{P}_{\widetilde{Y}}^{\bmod Z}=\mathcal{P}_{\widetilde{X}}^{\bmod D}$ for the $C^{\infty}$-functions with moderate growth for arguments in $U^{\text {an }}$ approaching the boundary. Computing $\boldsymbol{R} \widetilde{b}_{*}\left(\mathcal{A}_{\tilde{Y}}^{\text {? }}\right)$ via the flat resolutions (2.2) and (2.3) respectively, the assertions of the lemma follow immediately.

Now $\widetilde{\imath} \mathbb{C}_{U}$ an $[2 d]$ is the dualizing sheaf on the manifold $\widetilde{Y}$ with boundary $\widetilde{Y} \backslash U^{\text {an }}$ and similarly $\widetilde{\jmath}_{!} \mathbb{C}_{U \text { an }}[2 d]$ for $\widetilde{X}$. By Proposition 4.3 for the trivial connection $\left(\mathcal{O}_{Y}, d\right)$, we deduce from (2.2) the fine resolution

$$
\widetilde{\imath}_{!} \mathbb{C}_{U^{\text {an }}} \simeq\left(\mathcal{P}_{\tilde{Y}}^{<Z} \otimes_{\pi_{Y}^{-1}\left(C_{Y \text { an }}^{\infty}\right)} \pi_{Y}^{-1} \Omega_{Y \text { an }}^{\infty,(\cdot, \bullet)}, \partial, \bar{\partial}\right)
$$

where the right hand is to be understood as the simple complex associated to the indicated double complex of Dolbeault-type. Applying $\widetilde{b}_{*}$ thus yields an isomorphism

$$
\alpha: \boldsymbol{R} \widetilde{b}_{*} \widetilde{\imath}_{!} \mathbb{C}_{U^{\text {an }}}=\widetilde{b}_{*}\left(\mathcal{P}_{\widetilde{Y}}^{<Z} \otimes_{\pi_{Y}^{-1}\left(C_{Y^{\text {an }}}^{\infty}\right)} \pi_{Y}^{-1} \Omega_{Y^{\text {an }}}^{\infty,(\cdot, \cdot)}\right)=\widetilde{b}_{*} \mathcal{P}_{\widetilde{Y}}^{<Z} \otimes_{\pi_{X}^{-1}\left(C_{X^{\text {an }}}^{\infty}\right)} \pi_{X}^{-1} \Omega_{X^{\text {an }}}^{\infty,(\cdot,)} \cong \widetilde{\jmath}_{!} \mathbb{C}_{U^{\text {an }}}
$$

Since the isomorphisms of Lemma 4.5 had been constructed in the same way, the diagram

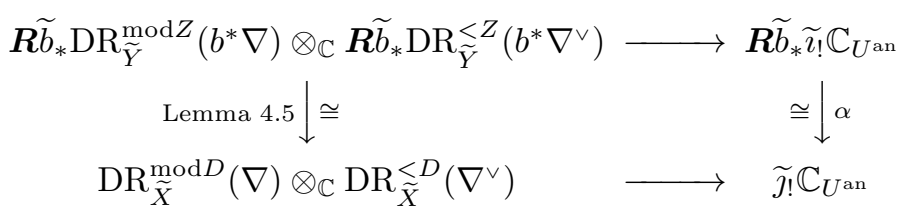


commutes. Hence, the morphism $\operatorname{DR}_{\widetilde{X}}^{\bmod D}(\nabla) \rightarrow \boldsymbol{R} \mathcal{H}$ om $\widetilde{X}_{\widetilde{X}}\left(\operatorname{DR}_{\tilde{X}}^{<D}\left(\nabla^{\vee}\right), \widetilde{\jmath}^{\mathbb{C}} \mathbb{C}_{U^{\text {an }}}\right)$ induced by the lower row of (4.8) factors as

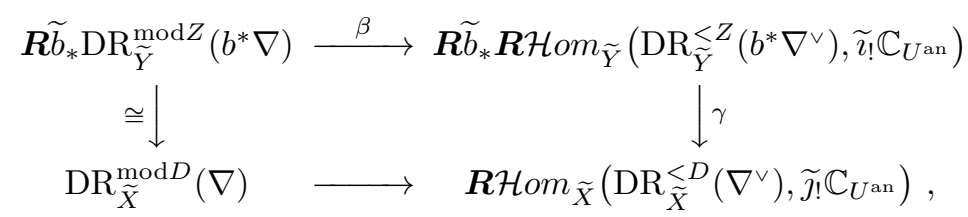

where $\gamma$ is given by the composition of the natural morphism

$$
\boldsymbol{R} \widetilde{\boldsymbol{b}}_{*} \boldsymbol{R} \mathcal{H o m}_{\widetilde{Y}}\left(\mathrm{DR}_{\widetilde{Y}}^{<Z}, \widetilde{\imath}_{!} \mathbb{C}_{U^{\text {an }}}\right) \rightarrow \boldsymbol{R} \mathcal{H}{ }_{\widetilde{X}}\left(\boldsymbol{R}_{*} \operatorname{DR}_{\widetilde{Y}}^{<Z}, \boldsymbol{R} \widetilde{b}_{*} \widetilde{l}_{!} \mathbb{C}_{U^{\text {an }}}\right)
$$

with the morphism $\alpha$ from above. By Poincaré-Verdier duality (Proposition 3.1.10 in [12]), $\gamma$ is an isomorphism. The same arguments apply in the case of the moderate de Rham complex. Consequently, in order to prove Theorem 4.4 namely the fact that the bottom row is an isomorphism, it suffices to do so for $\beta$.

In other words, we may a priori assume that $(E, \nabla)$ has a good lattice on $(X, D)$. Then we know by Proposition 4.3 that both complexes in the local duality pairing have cohomology in degree 0 only, i.e. we are reduced to prove that the pairing of sheaves

$$
\mathcal{S}^{\bmod D} \otimes{ }^{\vee} \mathcal{S}^{<D} \rightarrow \widetilde{\jmath}^{\mathbb{C}} \mathbb{C}_{U^{\text {an }}}
$$

is perfect, where $\mathcal{S}^{\bmod D}:=\mathcal{H}^{0}\left(\mathrm{DR}_{\widetilde{X}}^{\bmod D}\left(\nabla^{\vee}\right)\right)$ and ${ }^{\vee} \mathcal{S}^{<D}:=\mathcal{H}^{0}\left(\mathrm{DR}_{\widetilde{X}}^{<D}(\nabla)\right)$.

Exactly as in the proof of Proposition 4.3 due to the existence of a good lattice on $(X, D)$ and the asymptotic decomposition given by Theorem 3.2, we can reduce to the case where $\nabla$ is a rank one connection of the elementary form $\nabla=e^{\alpha} \otimes x^{\lambda}$ for some $\alpha \in \mathcal{O}_{X}(* D)$ and $\lambda \in \mathbb{C}^{d}$.

We restrict to a small enough open polysector $V \subset \widetilde{X} \stackrel{\pi}{\rightarrow} X$ where locally $D=\left\{x_{1} \cdots x_{k}=0\right\}$. We will write $\alpha(x)=x_{1}^{-m_{1}} \cdot x_{k}^{-m_{k}} \cdot u(x)$ with non-vanishing $u(x)$. Let us define the Stokes multi-directions of $\alpha$ along $D$ inside $V$ to be

$$
\Sigma_{\alpha}:=S t^{-1}\left(\left(\frac{\pi}{2}, \frac{3 \pi}{2}\right)\right),
$$

where $S t: \widetilde{D} \cap V \rightarrow \mathbb{R} / 2 \pi \mathbb{Z}$ denotes the map

$$
\operatorname{St}\left(r_{i}, \vartheta_{i}\right):=-\sum_{i=1}^{k} m_{i} \vartheta_{i}+\arg \left(u \circ \pi\left(r_{i}, \vartheta_{i}\right)\right) .
$$

Let $V_{\alpha}:=(V \backslash \widetilde{D}) \cup \Sigma_{\alpha}$, i.e. $V_{\alpha} \cap \widetilde{D}$ are the directions in which $e^{\alpha(x)}$ has rapid decay for $x$ radially approaching $D$. If $\widetilde{\jmath}_{\alpha}: V_{\alpha} \hookrightarrow V$ denotes the inclusion, one obviously has (possibly after shrinking $V$ ):

$$
\left.\mathcal{S}^{\bmod D}\right|_{V}=\left.\widetilde{\jmath}_{-\alpha}\left(e^{-\alpha(x)} \cdot \mathbb{C}_{U^{\text {an }}}\right)\right|_{V} \quad \text { and }\left.\quad{ }^{v} \mathcal{S}^{<D}\right|_{V}=\left.\widetilde{\jmath}_{\alpha}\left(e^{\alpha(x)} \cdot \mathbb{C}_{U^{\text {an }}}\right)\right|_{V} .
$$

Consequently we have the following commutative diagramm:

$$
\begin{aligned}
\left.\boldsymbol{R H o m}\left({ }^{\vee} \mathcal{S}^{<D}, \widetilde{\jmath}_{!} \mathbb{C}_{U^{\text {an }}}\right)\right|_{V} & \left.\longrightarrow \mathcal{S}^{\bmod D}\right|_{V} \\
\cong \downarrow & \downarrow \cong \\
\left.\boldsymbol{R H} \mathcal{H o m}\left(\left(\widetilde{\jmath}_{-\alpha}\right) ! \mathbb{C}_{V \backslash D}, \widetilde{\jmath}_{!} \mathbb{C}_{V \cap U^{\text {an }}}\right)\right|_{V} & \longrightarrow\left(\widetilde{\jmath}_{\alpha}\right) ! \mathbb{C}_{V_{\alpha}}
\end{aligned}
$$

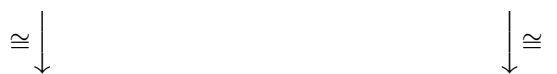

By the factorization $\widetilde{\jmath}=\widetilde{\jmath}_{-\alpha} \circ \widetilde{\iota}_{-\alpha}$ with $\widetilde{\iota}_{-\alpha}: V \cap U^{\text {an }} \hookrightarrow V_{-\alpha}$, we see that

$$
\begin{aligned}
& \boldsymbol{R H o m}\left(\left(\widetilde{\jmath}_{-\alpha}\right) ! \mathbb{C}_{V_{-\alpha}}, \widetilde{\jmath}_{!} \mathbb{C}_{U^{\text {an }}}\right) \cong\left(\widetilde{\jmath}_{-\alpha}\right)_{*} \boldsymbol{R} \mathcal{H o m}\left(\mathbb{C}_{V_{-\alpha}},\left(\widetilde{\iota}_{-\alpha}\right) ! \mathbb{C}_{V \cap U^{\text {an }}}\right) \cong \\
& \cong\left(\widetilde{\jmath}_{-\alpha}\right)_{*} \mathcal{H} \operatorname{Hom}\left(\mathbb{C}_{V_{-\alpha}},\left(\widetilde{\iota}_{-\alpha}\right) ! \mathbb{C}_{V \cap U^{\text {an }}}\right)=\left(\widetilde{\jmath}_{-\alpha}\right) ! \mathbb{C}_{V_{\alpha}},
\end{aligned}
$$

since $\left(V \backslash V_{\alpha}\right) \cap \widetilde{D}$ coincides with the closure of $V_{-\alpha} \cap \widetilde{D}$ inside $\widetilde{D}$. Hence, the bottom line of (4.12) is an isomorphism and thus

$$
\mathcal{S}^{\bmod D} \cong \boldsymbol{R} \mathcal{H} \operatorname{om}_{\tilde{X}}\left({ }^{\vee} \mathcal{S}^{<D}, \widetilde{\jmath}^{\mathfrak{I}} \mathbb{C}_{U^{\text {an }}}\right)
$$


locally on $\tilde{X}$ over an arbitrary point of $D$. Interchanging ${ }^{\vee} \mathcal{S}^{<D}$ and $\mathcal{S}^{\bmod D}$ gives the analogous isomorphism.

This completes the proof of the local duality result, Theorem 4.4 .

\section{Period integrals}

\subsection{Definition of rapid decay homology}

We now want to interpret the local duality pairing as a pairing via period integrals. To this end, let us recall the definition of rapid decay homology in [10.

Note that for the mere definition in [10, we consider the following geometric set-up. We start with the given flat algebraic connection $(E, \nabla)$ on the smooth quasi-projective variety $U$. We then compactify $U$ by some smooth projective $X$ with a normal crossing divisor $D:=X \backslash U$ as complement. Due to Mochizuki's Theorem, quoted as Theorem 3.1 above, we can pull-back the connection with respect to some regular birational map $b: Y \rightarrow X$ and obtain a good lattice for the connection. In other words, by replacing $X$ with $Y$ as a different choice of compactification, we may assume that the given connection admits a good lattice on the chosen compactification $(X, D)$.

Let $\pi: \widetilde{X} \rightarrow X$ denote the real oriented blow-up of the normal crossing divisor $D \subset X$. We will write $\widetilde{D}:=\pi^{-1}(D)$ and denote by $j: U \rightarrow X$ and $\widetilde{\jmath}: U \rightarrow \widetilde{X}$ the inclusions. For any $p \in \mathbb{N}_{0}$, we will write $S_{p}(\tilde{X})$ for the free $\mathbb{Q}$-vector space over all piecewise smooth maps $c: \Delta^{p} \rightarrow \widetilde{X}$ and we will consider singular homology with $\mathbb{Q}$-coefficients and piecewise smooth chains in the following.

Let $\mathcal{C}_{\widetilde{X}, \widetilde{D}}^{-p}$ denote the sheaf of relative $p$-chains of the pair $\widetilde{D} \subset \widetilde{X}$, i.e. the sheaf associated to the presheaf

$$
V \mapsto S_{p}(\widetilde{X},(\widetilde{X} \backslash V) \cup \widetilde{D})
$$

Let $\mathcal{E}:=\operatorname{ker}\left(\left.\nabla\right|_{U}\right)$ be the local system on $U$ of flat sections of $\nabla$. According to [10, Definition 2.3, a local section $c \otimes \varepsilon \in \Gamma\left(V, \mathcal{C}_{\widetilde{X}, \widetilde{D}}^{-} \otimes_{\mathbb{Q}} \widetilde{J}_{*} \mathcal{E}\right)$ is called a rapid decay chain, if for any point $y \in c\left(\Delta^{p}\right) \cap \widetilde{D} \cap V$ the following holds: Let $x_{1}, \ldots, x_{d}$ be local coordinates of $X$ around $y=0$ such that $D=\left\{x_{1} \cdots x_{k}=0\right\}$ for some $1 \leq k \leq d$. We chose a meromorphic basis $e: E \cong\left(\mathcal{O}_{X}(* D)\right)^{r}$ of $E$ at $y$ and require that if we develop $\varepsilon=\sum_{i=1}^{d} f_{i} \cdot e_{i}$ in this basis with analytic functions $f_{i} \in j_{*} \mathcal{O}_{U}$, these coefficients $f_{i}(x)$ decrease faster than any monomial for $x$ approahcing $D$, i.e. that for all $N \in \mathbb{N}^{k}$ there is a $C_{N}>0$ such that for all small enough $x$ we have

$$
\left|f_{i}(x)\right| \leq C_{N} \cdot\left|x_{1}\right|^{N_{1}} \cdots\left|x_{k}\right|^{N_{k}} .
$$

For chains $c \otimes \varepsilon$ inside $U$, we do not impose any condition. The sheaves of all these rapid decay $p$ chains will be denoted by $\mathcal{C}_{\widetilde{X}}^{\text {rd, }}-p(\nabla)$. Together with the usual boundary operator $\partial$ of singular chains they define the complex of rapid decay chains $\mathcal{C}_{\widetilde{X}}^{\text {rd }}(\nabla)$. The rapid decay homology of $(E(* D), \nabla)$ is the hypercohomology

$$
H_{k}^{r d}\left(U^{\mathrm{an}}, E, \nabla\right):=\mathbb{H}^{-k}\left(\widetilde{X}, \mathcal{C}_{\widetilde{X}}^{\mathrm{rd}}(\nabla)\right),
$$

which can be computed as the cohomology of the global sections, since the usual barycentric subdivision operator can be defined on the rapid decay chains and thus one deduces that the complex of rapid decay chains, similar to the sheaf complex of singular chains (cp. 27, p. 87) is homotopically fine. For more details, we refer to [10, section 2.3 .

\subsection{The local duality pairing and periods}

In order to obtain the desired interpretation of the local duality pairing (4.1) in terms of period integrals, we will examine the relation between the asymptotically flat de Rham complex of the dual connection $\left(E^{\vee}, \nabla^{\vee}\right)$ and its rapid decay complex. Let $\mathcal{E}^{\vee}$ denote the local system of the dual connection.

Recall that we chose $(X, D)$ in a way such that $(E, \nabla)$ admits a good lattice on $(X, D)$. According to Proposition 4.3, the natural inclusion

$$
\operatorname{DR}_{\tilde{X}}^{<D}\left(\nabla^{\vee}\right) \stackrel{\sim}{\longleftarrow} \mathcal{H}^{0}\left(\operatorname{DR}_{\tilde{X}}^{<D}\left(\nabla^{\vee}\right)\right)=: \mathcal{S}^{<D}
$$


is a quasi-isomorphism in this situation. Note that by definition and the properties of $\mathcal{A}_{\widetilde{X}}^{<D}$, the sheaf ${ }^{\vee} \mathcal{S}^{<D}$ consists of local solutions of $\nabla^{\vee}$ which have rapid decay along $D$. More precisely, given an open subset $V \subset \widetilde{X}$ and a section $\sigma \in \Gamma\left(V v^{\vee} \mathcal{S}^{<D}\right)$, the section $\sigma \in \mathcal{V}^{<D}(V) \subset \widetilde{\jmath}_{*}\left(\mathcal{E}^{\vee}\right)$ is rapidly decaying along any chain in $c \in \mathcal{C}_{\widetilde{X}, \widetilde{D}}^{-\cdot}(V)$, i.e.

$$
c \otimes \sigma \in \Gamma\left(V, \mathcal{C}_{\widetilde{X}}^{\mathrm{rd}}\left(\nabla^{\vee}\right)\right)
$$

Thus, we have a natural morphism

$$
\mathcal{C}_{\widetilde{X}, \widetilde{D}}^{-\cdot} \otimes_{\mathbb{Q}}{ }^{\vee} \mathcal{S}^{<D} \longrightarrow \mathcal{C}_{\widetilde{X}}^{\mathrm{rd}}\left(\nabla^{\vee}\right)
$$

Now, by standard arguments (e.g. [29]), the complex $\mathcal{C}_{\widetilde{X}, \widetilde{D}}$ is a homotopically fine resolution of the constant sheaf $\mathbb{C}_{\widetilde{X}}[2 d]$ : to see this, note first that $U:=\widetilde{X} \backslash \widetilde{D}$ is a real manifold of dimension $2 d$ and since we take homology relative to the boundary $\widetilde{D}$ we deduce that for small enough $V \subset \widetilde{X}$ such that $V \subset \Omega$ for some contractible $\Omega \subset \widetilde{X}$ we have by excision:

$$
\Gamma\left(V, \mathcal{H}^{-p}\left(\mathcal{C}_{\widetilde{X}, \widetilde{D}}^{\cdot}\right)\right)=H_{p}(\Omega,(\Omega \backslash V) \cup \widetilde{D}) \cong \begin{cases}0 & \text { for } p \neq 2 d \\ \mathbb{C} & \text { for } p=2 d\end{cases}
$$

Consequently, we end up with a natural morphism

$$
{ }^{\vee} \mathcal{S}^{<D}[2 d] \stackrel{\simeq}{\longrightarrow} \mathbb{C}_{\widetilde{X}}[2 d] \otimes \mathcal{S}^{<D} \stackrel{\simeq}{\longrightarrow} \mathcal{C}_{\widetilde{X}, \widetilde{D}}^{-} \otimes{ }^{\vee} \mathcal{S}^{<D} \stackrel{(5.2)}{\longrightarrow} \mathcal{C}_{\widetilde{X}}^{\mathrm{rd}}\left(\nabla^{\vee}\right)
$$

Proposition 5.1. If $(E, \nabla)$ admits a good lattice on $(X, D)$, there is a natural isomorphism

$$
\mathcal{C}^{\mathrm{rd}}\left(\nabla^{\vee}\right) \cong \mathrm{DR}_{\widetilde{X}}^{<D}\left(\nabla^{\vee}\right)[2 d]
$$

in the derived category $D^{b}\left(\mathbb{C}_{\widetilde{X}}\right)$.

Proof. Due to (5.1), it remains to prove that (5.2) is a quasi-isomorphism. Restricted to $U$, this is clear since there is no rapid decay condition involved and the rapid decay homology is nothing but the usual singular homology with values in the local system $\mathcal{E}^{\vee}$ which coincides with the restriction $\left.{ }^{\vee} \mathcal{S}^{<D}\right|_{U}$ on $U$.

As for the situation locally around $\widetilde{D}$ : By assumption on the existence of a good lattice, there is a cyclic ramification map $\rho: Y \rightarrow X$ such that $\rho^{*} \nabla^{\vee}$ admits an unramified good lattice. Since (5.2) obviously is compatible with direct image by $\rho$, it is enough to prove the assertion on $Y$, i.e. we can assume without loss of generality that $\nabla^{\vee}$ itself has an unramified good lattice. Exactly as in the proof of Proposition 4.3, we can apply Mochizuki's lifting theorem, Theorem 3.2 above, and reduce the statement to the case $\nabla^{\vee}=e^{\mathfrak{a}} \otimes \mathcal{R}$ for some regular singular connection $\mathcal{R}$. Now, since the solutions of a regular singular connection are moderate (see [8]), we have

$$
\mathcal{S}^{<D}\left(e^{\mathfrak{a}} \otimes \mathcal{R}\right)=\mathcal{S}^{<D}\left(e^{\mathfrak{a}}\right) \otimes \widetilde{\jmath}_{*} \mathcal{R}
$$

with the obvious notation $\mathcal{S}^{<D}(\nabla):=\mathcal{H}^{0}\left(\mathrm{DR}_{\widetilde{X}}^{<D}(\nabla)\right)$ for a given connection $\nabla$.

Now, consider a small open polysector $V$ around some direction $\vartheta \in \pi^{-1}(y)$ with $y \in D$. Let $\Sigma_{\mathfrak{a}} \subset$ $\pi^{-1}(y)$ denote the set of Stokes-directions of $\mathfrak{a}$ as in (4.10).

We distinguish the following cases with respect to the direction $\vartheta$ : If $\vartheta \in \Sigma_{\mathfrak{a}}$ then for $V$ being a small enough polysector, we have $\left.\mathcal{S}^{<D}\left(e^{\mathfrak{a}}\right)\right|_{V}=\left.\widetilde{\jmath}_{!}\left(e^{\mathfrak{a}} \mathbb{C}_{U}\right)\right|_{V}$. For a smooth topological chain $c$ in $\widetilde{X}$, the local section $e^{\mathfrak{a}}$ will not have rapid decay along $c$ in $V$ as required by the definition unless the chain does not meet $\widetilde{D} \cap V$. Hence

$$
\left.\mathcal{C}_{\widetilde{X}}^{\mathrm{rd}}\left(e^{\mathfrak{a}}\right)\right|_{V}=\left.\mathcal{C}_{\widetilde{X}, \widetilde{D}}^{-\cdot} \otimes \mathcal{S}^{<D}\left(e^{\mathfrak{a}}\right)\right|_{V}
$$

If $\vartheta \in \Sigma_{\mathfrak{a}}$, we can assume that $V$ is an open polysector such that all the arguments of points in $V$ are contained in $\Sigma_{\mathfrak{a}}$. Then $\left.\mathcal{S}^{<D}\left(e^{\mathfrak{a}}\right)\right|_{V} \cong \widetilde{\jmath}_{*}\left(e^{\mathfrak{a}} \mathbb{C}_{V}\right)$. Similarly, all twisted chains $c \otimes e^{\mathfrak{a}}$ will have rapid decay inside $V$ and again both complexes considered are equal to $\mathcal{C}_{\widetilde{X}, \widetilde{D}}^{-\cdot} \otimes \widetilde{\jmath}_{*}\left(e^{\mathfrak{a}} \mathbb{C}_{U}\right)$.

Finally, if $\vartheta$ separates the Stokes regions of $e^{\mathfrak{a}}$ and $e^{-\mathfrak{a}}$, we have with the notation of (4.11):

$$
\left.\left.\mathcal{S}^{<D}\left(e^{\mathfrak{a}}\right)\right|_{V} \cong\left(\widetilde{\jmath}_{\mathfrak{a}}\right) !\left(e^{\mathfrak{a}} \mathbb{C}_{U}\right)\right|_{V} .
$$


The subspace $V_{\mathfrak{a}}$ is characterized by the property that $V_{\mathfrak{a}} \cap \widetilde{D}$ consists of those directions along which $e^{\mathfrak{a}(x)}$ has rapid decay for $x$ approaching $\widetilde{D}$. In particular, $c \otimes e^{\mathfrak{a}}$ is a rapid decay chain on $V$ if and only if the topological chain $c$ in $\widetilde{X}$ approaches $\widetilde{D} \cap V$ in $V_{\mathfrak{a}}$ at most. Hence

$$
\left.\mathcal{C}_{\widetilde{X}}^{\mathrm{rd}}\left(e^{-\mathfrak{a}}\right)\right|_{V}=\mathcal{C}_{\widetilde{X}, \widetilde{D}}^{-\cdot} \otimes\left(\widetilde{\jmath}_{\mathfrak{a}}\right) !\left(e^{\mathfrak{a}} \mathbb{C}_{U}\right)=\left.\mathcal{C}_{\widetilde{X}, \widetilde{D}}^{-\cdot} \otimes{ }^{\vee} \mathcal{S}^{<D}\right|_{V}
$$

Thus, we have seen that (5.2) is a quasi-isomorphism in all three cases.

We remain in the situation given above, namely $(E, \nabla)$ admitting a good lattice on $(X, D)$. Due to Proposition 4.3 the local duality pairing reduces to the pairing

$$
{ }^{\vee} \mathcal{S}^{<D} \otimes \mathcal{S}^{\bmod D} \longrightarrow \widetilde{\jmath} \mathbb{C}_{U}
$$

given by multiplication.

In order to be able to apply Proposition 5.1, consider the resolution

$$
\mathcal{A}_{\widetilde{X}}^{<D} \otimes_{\pi^{-1} \mathcal{O}_{X}} \pi^{-1} \Omega_{X}^{r} \hookrightarrow\left(\mathcal{P}_{\widetilde{X}}^{<D} \otimes_{\pi^{-1} C_{X}^{\infty}} \pi^{-1} \Omega_{X^{\text {an }}}^{\infty,(r, \bullet)}, \bar{\partial}\right)
$$

induced by the resolution (2.2), where $\Omega_{X^{\text {an }}}^{\infty,(r, s)}$ denotes the sheaf of $C^{\infty}$ forms on $X$ of degree $(r, s)$. Let $\mathcal{R} d \cdot$ denote the total complex associated to the bicomplex

$$
\mathcal{R} d^{\bullet, \bullet}:=\left(\mathcal{P}_{\widetilde{X}}^{<D} \otimes_{\pi^{-1} C_{X}^{\infty}} \pi^{-1} \Omega_{X^{\text {an }}}^{\infty,(\bullet \bullet)}, \partial, \bar{\partial}\right) .
$$

Then, $\mathcal{R} d \cdot$ is a fine resolution of $\widetilde{\jmath}_{!} \mathbb{C}_{U}$.

Next, consider the canonical quasi-isomorphism $\beta: \mathcal{C}_{\widetilde{X}, \widetilde{D}}^{-\cdot} \otimes \mathcal{R} d \stackrel{\simeq}{\longrightarrow} \mathfrak{D b}_{\widetilde{X}}^{\text {rd, }}{ }^{-\cdot}$ which maps an element $c \otimes \rho \in \mathcal{C}_{\widetilde{X}, \widetilde{D}}^{-r} \otimes \mathcal{R} d^{s}(V)$ of the left hand side over some open $V \subset \widetilde{X}$ to the distribution given by $\eta \mapsto \int_{c} \eta \wedge \rho$ for a test form $\eta$ with compact support in $V$. Because of the rapid decay property of $\rho$, this distribution has the property expressed in (2.4), hence is indeed a local section of $\mathfrak{D b}_{\widetilde{X}}^{\text {rd,s-r }}$.

Now, the main step towards the interpretation as period integrals is to define the following pairing:

$$
\gamma: \mathcal{C}_{\widetilde{X}}^{\mathrm{rd},-r}\left(\nabla^{\vee}\right) \otimes \mathrm{DR}_{\widetilde{X}}^{\bmod D, s}(\nabla) \rightarrow \mathfrak{D b}_{\widetilde{X}}^{\mathrm{rd}, s-r},(c \otimes \varepsilon, \omega) \mapsto\left(\eta \mapsto \int_{c} \eta \wedge\langle\varepsilon, \omega\rangle\right),
$$

where we observe that since $\varepsilon$ is rapidly decaying along $c$ and $\omega$ is of moderate growth at most, the integral first of all converges and it satisfies the estimate (2.4) for all test forms $\eta$ with compact support on $\widetilde{X}$. The resulting pairing (5.4) is called the period pairing of the connection $\nabla$.

Now, the inclusion $\mathcal{S}^{\bmod D} \rightarrow \mathrm{DR}_{\widetilde{X}}^{\bmod D}(\nabla)$ is a quasi-isomorphism and the period pairing and the local duality pairing (in the form of (5.3) and with the appropriate shift) fit into the commutative diagramm

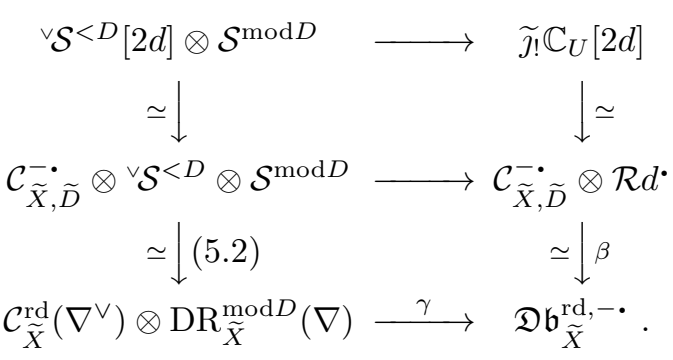

Applying the local duality result, Theorem 4.4 together with Poincaré-Verdier duality gives an isomorphism

$$
\boldsymbol{R} \Gamma\left(\mathrm{DR}_{\widetilde{X}}^{<D}\left(\nabla^{\vee}\right)[2 d]\right) \cong \boldsymbol{R} \Gamma \boldsymbol{R} \mathcal{H}_{\mathbb{C}_{\widetilde{X}}}\left(\operatorname{DR}_{\widetilde{X}}^{\bmod D}(\nabla), \widetilde{\jmath}_{!} \mathbb{C}_{U}[2 d]\right) \cong \operatorname{Hom}_{\mathbb{C}}\left(\boldsymbol{R} \Gamma\left(\mathrm{DR}_{\widetilde{X}}^{\bmod D}(\nabla)\right), \mathbb{C}\right)
$$

In summary, we have obtained a description of the perfect duality

$$
\mathbb{H}^{2 d-p}\left(\widetilde{X}, \mathrm{DR}_{\widetilde{X}}^{<D}\left(\nabla^{\vee}\right)\right) \otimes \mathbb{H}^{p}\left(\widetilde{X}, \mathrm{DR}_{\tilde{X}}^{\bmod D}(\nabla)\right) \longrightarrow \mathbb{C}
$$

induced by the local duality pairing, in terms of the period pairing (5.4). We summarize this result: 
Theorem 5.2. The period pairing (5.4) induces a perfect pairing

$$
H_{p}^{r d}\left(U, E^{\vee}, \nabla^{\vee}\right) \otimes_{\mathbb{C}} H_{d R}^{p}(U ; E, \nabla) \longrightarrow \mathbb{C}
$$

betweem the algebraic de Rham cohomology of $(E, \nabla)$ and the rapid decay homology of the dual connection.

\subsection{The period determinant}

Let $k \subsetneq \mathbb{C}$ be a subfield of the field of complex numbers and assume that the given geometric data, the variety $U$ as well as the vector bundle and the connection are defined over $k$ already. Then the de Rham cohomology inherits this $k$-structure, i.e. $H_{d R}(U, E, \nabla)$ is naturally a $k$-vector space.

Now, let $F \subsetneq \mathbb{C}$ denote another subfield of $\mathbb{C}$ and let us assume that the local system $\mathcal{E}$ on $U^{a n}$ comes equipped with a given $F$-structure. In analogy to [26] (for the regular singular case), we consider the category $W_{k, F}(U)$ - which we already defined in [10], 2.5 - of triples $\mathcal{M}=\left((E, \nabla), \mathcal{E}_{F}, \rho\right)$ with:

i) a vector bundle $E$ on $U$ with rank $r$ together with a flat connection $\nabla: E \rightarrow E \otimes_{\mathcal{O}_{U}} \Omega_{U}^{1}$,

ii) a local system $\mathcal{E}_{F}$ of $F$-vector spaces on the analytic manifold $U^{a n}$,

iii) a morphism $\rho: \mathcal{E}_{F} \rightarrow E^{a n}$ of sheaves on $U^{a n}$ inducing an isomorphism $\mathcal{E}_{F} \otimes_{F} \mathbb{C} \stackrel{\sim}{\rightarrow} \operatorname{ker}\left(\nabla^{a n}\right)$ of local systems of $\mathbb{C}$-vector spaces on $U^{a n}$.

A morphism between $\left((E, \nabla), \mathcal{E}_{F}, \rho\right)$ and $\left(\left(E^{\prime}, \nabla^{\prime}\right), \mathcal{E}_{F}{ }^{\prime}, \rho^{\prime}\right)$ is given by a morphism $E \rightarrow E^{\prime}$ respecting the connections together with a morphism $\mathcal{E}_{F} \rightarrow \mathcal{E}_{F}{ }^{\prime}$ of $F$-local systems with the natural compatibility condition with respect to $\rho$ and $\rho^{\prime}$.

Let $\left((E, \nabla), \mathcal{E}_{F}, \rho\right) \in W_{k, F}(U)$ be an object in this category for given subfields $k, F \subsetneq \mathbb{C}$. Then the local system $\mathcal{E}^{\vee}$ inherits an $F$-structure from the given $F$-structure on $\mathcal{E}$. Consequently, we can consider the $F$-lattice

$$
\mathcal{C}_{\widetilde{X}}^{\mathrm{rd},-p}\left(\nabla^{\vee}\right)_{F} \subset \mathcal{C}_{\widetilde{X}, \widetilde{D}}^{-p} \otimes_{\mathbb{Q}} \widetilde{J}_{*} \mathcal{E}_{F}^{\vee}
$$

of all rapidly decaying chains in $\mathcal{C}_{\widetilde{X}, \widetilde{D}}^{-p} \otimes_{\mathbb{Q}} \widetilde{\jmath}_{*} \mathcal{E}_{F}^{\vee}$ and end up with a natural $F$-lattice $H_{p}^{r d}\left(U^{\text {an }}, E, \nabla\right)_{F}$ inside the rapid decay homology:

$$
H_{p}^{r d}\left(U^{\mathrm{an}}, E, \nabla\right)_{F} \otimes_{F} \mathbb{C} \stackrel{\cong}{\longrightarrow} H_{p}^{r d}\left(U^{\mathrm{an}}, E, \nabla\right),
$$

the isomorphism induced by $\rho$. The duality between the algebraic de Rham cohomology and the rapid decay homology via the period pairing enables us to compare these lattices and to generalize [10, Definition 2.7, unconditionally to the case of arbitrary dimension of $U$ :

Definition 5.3. For $\left((E, \nabla), \mathcal{E}_{F}, \rho\right) \in W_{k, F}(U)$, we define its period determinant to be the element

$$
\operatorname{det}\left((E, \nabla), \mathcal{E}_{F}, \rho\right):=\prod_{p \geq 0} \operatorname{det}\left(\left\langle\gamma_{j}^{(p)}, \omega_{i}^{(p)}\right\rangle\right)_{i, j}^{(-1)^{p}} \in \mathbb{C}^{\times} / k^{\times} F^{\times},
$$

where $\omega_{i}^{(p)}$ denotes a basis of $H_{d R}^{p}(U, E, \nabla)$ over $k$ and $\gamma_{j}^{(p)}$ a basis of the $F$-vector space $H_{k}^{r d}\left(U^{\mathrm{an}}, E^{\vee}, \nabla^{\vee}\right)_{F}$.

We hope that this will be the starting point of further examinations in the spirit of T. Saito and T. Terasoma's work [26] in the case of regular singular connections now for irreguar ones also.

\section{References}

[1] Y. André, Comparison theorems between algebraic and analytic De Rham cohomology (with emphasis on the p-adic case), J. de Théorie des Nombres de Bordeaux 16 (2004), 335 - 355

[2] A. Beilinson, S. Bloch, H. Esnault, $\varepsilon$-factors for Gauss-Manin determinants, Mosc. Math. J. 2(2002), no. 3, 477 - 532

[3] S. Bloch, H. Esnault, Homology for irregular connections, Journal de Théorie des Nombres de Bordeaux 16 (2004), 65 $-78$

[4] S. Bloch, H. Esnault, Gauss-Manin determinant connections and periods for irregular connections, GAFA 2000 (Tel Aviv 1999), Geom. Funct. Anal. 2000, Special Volume, Part I, 1 - 31

[5] S. Bloch, H. Esnault, Gauss-Manin determinants for rank 1 irregular connections on curves. With an appendix in French by P. Deligne, Math. Ann. 321(2001), no. 1, $15-87$

[6] A. Borel, Algebraic D-modules, Perspectives in Math. 2, Academic Press, Boston 1987

[7] G.E. Bredon, Sheaf Theory, Second Edition, Grad. texts in Math. 170, Springer-Verlag, Berlin Heidelberg, 1997

[8] P. Deligne, Equations différentielles à points singuliers réguliers, LNS 163, Springer-Verlag, Berlin Heidelberg, 1970 
[9] A. Grothendieck, On the de Rham cohomology of algebraic varieties, Publ. Math. IHES 29 (1966), $93-103$

[10] M. Hien, Periods for irregular singular connections on surfaces, Math. Ann. 337 (2007), 631 - 669

[11] L. Hörmander, The analysis of linear partial differential operators, I, Springer-Verlag, Berlin-Heidelberg, 1990

[12] M. Kashiwara, P. Schapira, Sheaves on manifolds, Grundlehren der mathem. Wissenschaft 292, Springer Verlag Berlin Heidelberg, 1990

[13] H. Majima, Asymptotic analysis for integrable connections with irregular singular points, LNS 1075, Springer-Verlag, Berlin, Heidelberg, 1984

[14] B. Malgrange, Equations différentielles à coefficients polynomiaux, Prog. in Math. 96, Birkhäuser, Basel, Boston, 1991

[15] B. Malgrange, Ideals of differentiable functions, Oxford University Press, 1966

[16] B. Malgrange, Connexions méromorphes II, le réseau canonique, Invent. Math. 124 (1996), 367 - 387

[17] Z. Mebkhout, Le théorème de comparaison entre cohomologies de de Rham d'une variété algébrique complexe et le théorème d'existence de Riemann, Publ.Math. IHES 69(1989), 47-89

[18] Z. Mebkhout, Le formalisme de six opérations de Grothendieck pour les $\mathcal{D}_{X}$-modules cohérents, Travaux en Cours 35 , Hermann, Paris, 1989

[19] T. Mochizuki, Good formal structure for meromorphic flat connections on smooth projective surfaces, Preprint, March 2008, arXiv:0803.1346 1 [math.AG]

[20] T. Mochizuki, Wild harmonic bundles and wild pure twistor D-modules, Preprint, March 2008, arXiv:0803.1344 1 [math.AG]

[21] L. Narváez Macarro, The local duality theorem in $\mathcal{D}$-module theory, in Eléments de la théorie des systèmes différentiells géométrique, Sémin. Congr. 8, Soc. Math. France, Paris, 2004

[22] J.-P. Ramis, Y. Sibuya, Hukuhara domains and fundamental existence and uniqueness theorems for asymptotic solutions of Gevrey type, Asymptotic Analysis 2 (1989), no.1, 39 - 94

[23] C. Sabbah, Equations différentielles à points singuliers irréguliers et phénomène de Stokes en dimension 2, Astérisque 263, Soc. Math. de France, 2000

[24] C. Sabbah, Equations différentielles à points singuliers irréguliers en dimension 2, Ann. Inst. Fourier (Grenoble) 43 (1993), $1619-1688$

[25] C. Sabbah, On the comparison theorem for elementary irregular $\mathcal{D}$-modules, Nagoya Math. J., 141 (1996), 107 - 124

[26] T. Saito, T. Terasoma, Determinant of period integrals, J. AMS 10 (1997), no. 4, 865 - 937

[27] R. G. Swan, The theory of sheaves, Chicago Lectures in Mathematics, The University of Chicago Press, Chicago/London, 1964

[28] T. Terasoma, Confluent hypergeometric functions and wild ramification, J. of Algebra 185 (1996), 1 - 18

[29] J.-L. Verdier, Classe d'homologie associée a un cycle, in 'Séminaire de géométrie analytique', Astérisque 36/37, 1976, $101-151$

Marco Hien, NWF I - Mathematik, Universität Regensburg, 93040 Regensburg, Germany

E-mail address: marco.hien@mathematik.uni-regensburg.de 